\title{
Efficacy of Different Management Practices against Chilo partellus (Swinhoe) in Kharif Maize Crop in Western Uttar Pradesh, India
}

\author{
Vishvendra, Sandeep Kumar*, Visvash Vaibhav, Ankush Kumar and D.V. Singh \\ S.V.P. University of Agriculture and Technology, Meerut- 250110, U.P., India \\ *Corresponding author
}

\begin{tabular}{|c|c|}
\hline $\begin{array}{l}\text { Ke y w or d s } \\
\text { Insecticide, Maize } \\
\text { stem borer. }\end{array}$ & $\begin{array}{l}\text { cent. Among all the treatments the seed treatment with Spinosad } 45 \% \text { SC spray @ } \\
\text { partellus ( } 82.95 \text { per cent reduction After First Spray (AFS), } 87.26 \text { per cent reduction After }\end{array}$ \\
\hline Article Info & rts ( 1.66 per cent at 30 DAS and 2.33 per cent at 45 DAS) and percent life injury (1.33 \\
\hline $\begin{array}{l}\text { Accepted: } \\
\text { 23 September } 2017 \\
\text { Available Online: } \\
10 \text { October } 2017\end{array}$ & $\begin{array}{l}\text { Second Spray (ASS) and } 78.17 \text { per cent reduction After Third Spray (AFS) per cent dead } \\
\text { hearts ( } 2.33 \text { per cent at } 30 \text { DAS and } 3.33 \text { per cent at } 45 \text { DAS) and percent life injury (1.66 }\end{array}$ \\
\hline & $\begin{array}{l}\text { spray @ } 250 \mathrm{ml} / \mathrm{ha}(58.50 \text { per cent reduction After First Spray (AFS), } 61.52 \text { per cent } \\
\text { reduction After Second Spray (ASS) and } 63.01 \text { per cent reduction After Third Spray (AFS) } \\
\text { per cent dead hearts }(7.33 \text { per cent at } 30 \mathrm{DAS} \text { and } 7.66 \text { per cent at } 45 \text { DAS) and percent } \\
\text { life injury }(6.20 \text { per cent at } 30 \text { DAS and } 6.66 \text { per cent at } 45 \text { DAS). The maximum grain } \\
\text { yield of } 45.1 \mathrm{q} / \mathrm{ha} \text { and net profit of Rs. } 24345 \text { /ha obtained from the treatment of Spinosad } \\
\text { and yield of } 38.44 \mathrm{q} / \mathrm{ha} \text { and net profit of Rs. } 22486 / \text { ha obtained from the treatment of } \\
\text { Bifenthrin. The minimum grain yield of } 28.41 \mathrm{q} / \mathrm{ha} \text { and net profit of Rs. } 7145 / \text { ha was } \\
\text { recorded in untreated control. }\end{array}$ \\
\hline
\end{tabular}

\section{Introduction}

Maize or corn (Zea mays Linn.) is one of the important cereal crops of the world, cultivated for food, fodder and for raw material in many industries. In many parts of the world, it is an important food crop. Maize grain contains about 10 percent protein, 4 percent oil, 70 percent carbohydrates, 2-3 per cent crude fiber, 10.4 percent albuminoides, 1.4 percent ash. Its protein "ZEIN" deficient in two essential amino acids, tryptophan and lysine. 
It also contains significant quantities of vitamin E calcium \& phosphorus. Around 250 insect and mite species are attacking different stages of maize. The average losses caused by the insects are estimated around 10\%. Among them, stem borer, Chilo partellus (Swinhoe), is the most serious pest during kharif causing 26.7-80.04\% yield loss in different agro climatic regions of India.

Maize stem borer, Chilo partellus Swinhoe (Lepidoptera: Pyralidae) is one of the major biotic constraints in successful maize and sorghum production worldwide (James, 2003), particularly in Asia and Africa (Siddiqui \& Marwaha, 1993). It has been reported to cause severe losses in maize crop throughout its geographical distribution including India. Yield losses of $24-75 \%$ have been reported by the attack of this pest alone.

\section{Materials and Methods}

To evaluate the efficacy of insecticides to control maize stem borer, the experiment was conducted at Crop research center, (C.R.C.) of the S.V.P.U.A\&T, Meerut in western Uttar Pradesh. There were six insecticidal treatments, along with control. The experiment was conducted in a Randomized Block Design (R.B.D.) with seven treatments and replicated thrice. Ten plants were selected randomly for each plot. The total number of plots was twenty one. The maize seeds of cultivar 'SUJATA' were sown in plots size 4 x $3 \mathrm{~m}^{2}$.

The dead hearts and leaf injury rating due to attack of stem borer were counted from randomly selected ten plants, at 30 and 45 DAS and their percentage was calculated on the basis of total plants observed. These plants were observed one day before and seven days after application. In treated plots insecticides application was done thrice during the crop season. First application of insecticides was given at 15 days after sowing, second application of insecticides was done after 30 days after sowing and third application was done after 45 days of sowing. The grain yields of all the plots were recorded at the time of harvest. Finally, the data obtained were subjected for arc sin transformation and then statistically analyzed.

\section{Results and Discussion}

Pretreatment count of stem borer per ten plants ranged from 11.66 to 17.78 per cent. The stem borer population reduced among all the treatments on different days of observation (Table 1).

\section{Application of insecticides}

\section{After first application of insecticides}

All the treatments were found effective and significantly superior to control treatment. Spinosad 45\% S.C. @ $200 \mathrm{ml} /$ ha was found most effective insecticide with (82.95\%) reduction of stem borer incidence. It was significantly superior with other all over treatments, followed by Bifenthrin $10 \%$ E.C @ $400 \mathrm{ml} / \mathrm{ha}(75.92 \%)$. The least reduction of stem borer incidence was recorded in the plots treated with Imidacloprid $17.8 \%$ SL (58.50\%) insecticide (Table 1).

\section{After second application insecticides}

The second insecticidal application was done after thirty days of sowing. The data on mean per cent reduction over untreated control was revealed that all the treatments were effective and significantly superior to the untreated control. Spinosad 45 \% S.C. @ 200 ml/ha was found the most effective treatments and reduced $(87.26 \%)$ stem borer incidence followed by Biffenthrin 10\% E.C. @ 400 $\mathrm{ml} /$ ha reduced $(80.59 \%)$. Table data revealed that order of effectiveness of different 
insecticides after second application as follows, Cartap hydrochloride 50 \% S.P. @ 1Kg./ha (77.56 \%), Carbofuran 3G @ 20 Kg/ha (74.23\%), Phorate 10G @ 15 K.g /ha (70.91\%) and Imidachloprid 17.8\% SL @ $250 \mathrm{ml} /$ ha reduced $(61.52 \%)$ (Table 1$)$.

\section{After third application of insecticides}

The third application was done at forty five days after sowing. The data on mean per cent reduction over untreated control revealed that all the treatments were effective and significantly superior over untreated control. Spinosad 45\% S.C. @ $200 \mathrm{ml} / \mathrm{ha}$ was the found most effective treatment with $(87.57 \%)$ reduction of stem borer incidence. Bifenthrin $10 \%$ E.C. @ 400 ml/ha reduced $(81.20 \%)$ stem borer infestation but it not differ significantly Cartap hydrochloride $50 \%$ S.P. @ $1 \mathrm{~kg} / \mathrm{ha}$ reduced $(78.17 \%)$ stem borer infestation after 7 days after treatment. Carbofuran3 G @ 20 kg/ha and Phorate 10 G @ $15 \mathrm{~kg} / \mathrm{ha}$ had larvae incidence $(75.14 \%)$ and $(71.82 \%)$ and were at par with each other. Imidachloprid 17.8\% SL @ 250 ml/ha was least effective with the lowest (63.06 \%) stem borer incidence (Table 1).

Similarly, Ahmed et al., (2002) who reported that Spinosad $45 \%$ SC was very effective and recorded maximum reduction of the stem borer population.

\section{Dead hearts}

\section{Thirty days after sowing}

The data on dead heart formation is presented in table 1 . The minimum dead heart formation (1.66) was found in the plots treated with Spinosad 45\% S.C. @ $200 \mathrm{ml} / \mathrm{ha}$ followed by Bifenthrin $10 \%$ E.C. @ 400 ml/ha (2.33), Cartap hydrochloride $50 \%$ S.P. @ 1 kg/ha (3.33) and Carbofuran 3 G @ 20 kg/ha (4.66) per cent dead hearts formation. The more dead heart were found in the Phorate $10 \mathrm{G} @$ $15 \mathrm{~kg} / \mathrm{ha}(6.33)$ and Imidachloprid $17.8 \%$ SL@ $250 \mathrm{ml} / \mathrm{ha}$ (7.33) treated plots. Maximum dead hearts was recorded in untreated control $(8.33 \%)$ (Table 1$)$.

The treatment details for the management of maize stem borer were as follows

\begin{tabular}{|c|c|c|c|c|}
\hline \multirow{2}{*}{$\begin{array}{c}\text { Symbol of } \\
\text { Treatments }\end{array}$} & \multicolumn{2}{|l|}{ Treatments } & \multirow[t]{2}{*}{ Dose } & \multirow{2}{*}{$\begin{array}{l}\text { Methods of } \\
\text { Application }\end{array}$} \\
\hline & Chemical name & Trade name & & \\
\hline $\mathrm{T}_{1}$ & Spinosad $45 \%$ SC & Tracer & $200 \mathrm{ml} / \mathrm{ha}$ & Spraying \\
\hline $\mathrm{T}_{2}$ & Phorate $10 \mathrm{G}$ & Phorate & $15 \mathrm{~kg} / \mathrm{ha}$ & Broadcasting \\
\hline $\mathrm{T}_{3}$ & Carbofuran $3 \mathrm{G}$ & Crown & $20 \mathrm{~kg} / \mathrm{ha}$ & Broadcasting \\
\hline $\mathrm{T}_{4}$ & Bifenthrin $10 \% \mathrm{EC}$ & Talstar & $400 \mathrm{ml} / \mathrm{ha}$ & Spraying \\
\hline $\mathrm{T}_{5}$ & Cartap hydrochloride $50 \%$ SP & Cargo & $1 \mathrm{~kg} / \mathrm{ha}$ & Spraying \\
\hline $\mathrm{T}_{6}$ & Imidacloprid $17.8 \% \mathrm{SL}$ & Jubilant & $250 \mathrm{ml} / \mathrm{ha}$ & Spraying \\
\hline $\mathrm{T}_{7}$ & Control & ---- & - & $\begin{array}{c}\text { Water } \\
\text { spraying }\end{array}$ \\
\hline
\end{tabular}


Table.1 Efficacy of insecticides against maize stem borer chilo pertellus

\begin{tabular}{|c|c|c|c|c|c|c|c|c|c|c|}
\hline \multirow[b]{2}{*}{ Treatment } & \multirow[t]{2}{*}{ Dose } & \multirow{2}{*}{$\begin{array}{c}\text { Pre } \\
\text { treatment } \\
(\%)\end{array}$} & \multicolumn{3}{|c|}{ Mean per cent reduction $(\%)$} & \multirow[t]{2}{*}{ Mean } & \multicolumn{2}{|c|}{ Per cent DH } & \multicolumn{2}{|c|}{ Per cent LIR } \\
\hline & & & $\begin{array}{c}\text { After } \\
1^{\text {st }} \\
\text { spray }\end{array}$ & $\begin{array}{c}\text { After } \\
\text { 2nd } \\
\text { spray }\end{array}$ & $\begin{array}{c}\text { After } \\
3^{\text {rd }} \\
\text { spray }\end{array}$ & & $\begin{array}{c}30 \\
\text { DAS }\end{array}$ & $\begin{array}{c}45 \\
\text { DAS }\end{array}$ & $\begin{array}{c}30 \\
\text { DAS }\end{array}$ & $\begin{array}{l}45 \\
\text { DAS }\end{array}$ \\
\hline Spinosad $45 \%$ Sc & $\begin{array}{c}200 \\
\mathrm{ml} / \mathrm{ha}\end{array}$ & $\begin{array}{c}15.57 \\
(23.12)\end{array}$ & $\begin{array}{c}82.95 \\
(65.76)\end{array}$ & $\begin{array}{c}87.26 \\
(69.26)\end{array}$ & $\begin{array}{c}87.57 \\
(69.56)\end{array}$ & 85.93 & 1.66 & 2.33 & 1.33 & 1.66 \\
\hline Phorate 10G & $\begin{array}{c}15 \\
\mathrm{~kg} / \mathrm{ha}\end{array}$ & $\begin{array}{c}13.34 \\
(20.97)\end{array}$ & $\begin{array}{c}68.89 \\
(56.08)\end{array}$ & $\begin{array}{c}70.91 \\
(57.34)\end{array}$ & $\begin{array}{c}71.82 \\
(57.92)\end{array}$ & 70.54 & 6.33 & 7.00 & 5.44 & 5.66 \\
\hline Carbofuran3G & $\begin{array}{c}20 \\
\mathrm{~kg} / \mathrm{ha}\end{array}$ & $\begin{array}{c}11.12 \\
(15.98)\end{array}$ & $\begin{array}{c}72.59 \\
(58.46)\end{array}$ & $\begin{array}{c}74.23 \\
(59.55)\end{array}$ & $\begin{array}{c}75.14 \\
(60.12)\end{array}$ & 73.98 & 4.66 & 5.00 & 4.33 & 4.66 \\
\hline Bifenthrin 10\% EC & $\begin{array}{c}400 \\
\mathrm{ml} / \mathrm{ha}\end{array}$ & $\begin{array}{c}15.56 \\
(22.09)\end{array}$ & $\begin{array}{c}75.92 \\
(60.50)\end{array}$ & $\begin{array}{l}80.59 \\
(63.84)\end{array}$ & $\begin{array}{c}81.20 \\
(64.18)\end{array}$ & 79.24 & 2.33 & 3.33 & 1.66 & 2.33 \\
\hline $\begin{array}{l}\text { Cartap } \\
\text { hydrochloride } \mathbf{5 0 \%} \\
\text { SP }\end{array}$ & $\begin{array}{c}1 \\
\mathrm{Kg} / \mathrm{ha}\end{array}$ & $\begin{array}{c}13.34 \\
(24.89)\end{array}$ & $\begin{array}{c}75.53 \\
(60.61)\end{array}$ & $\begin{array}{l}77.56 \\
(61.78)\end{array}$ & $\begin{array}{c}78.17 \\
(62.56)\end{array}$ & 77.09 & 3.33 & 3.66 & 2.78 & 3.66 \\
\hline $\begin{array}{l}\text { Imidachloprid17.8\% } \\
\text { SL }\end{array}$ & $\begin{array}{c}250 \\
\mathrm{ml} / \mathrm{ha}\end{array}$ & $\begin{array}{c}17.78 \\
(15.98)\end{array}$ & $\begin{array}{c}58.50 \\
(49.88)\end{array}$ & $\begin{array}{c}61.52 \\
(51.64)\end{array}$ & $\begin{array}{c}63.06 \\
(52.56)\end{array}$ & 61.03 & 7.33 & 7.66 & 6.20 & 6.66 \\
\hline Control & --- & $\begin{array}{c}11.12 \\
(15.34)\end{array}$ & 0.00 & 0.00 & 0.00 & 0.00 & 8.33 & 9.33 & 7.33 & 8.66 \\
\hline SE (m) & & NS & 1.89 & 1.39 & 1.487 & 1.589 & 1.73 & 2.37 & 2.02 & 2.51 \\
\hline$C D(p=0.005)$ & & & 5.902 & 4.34 & 4.632 & 4.958 & 0.55 & 0.76 & 0.65 & 0.79 \\
\hline
\end{tabular}

Table.2 Economics of various treatments against C. partellus during Kharif, 2014

\begin{tabular}{|c|c|c|c|c|c|c|c|c|}
\hline $\begin{array}{l}\text { Treatment } \\
\text { No. }\end{array}$ & Treatment Name & Dose/ha & $\begin{array}{l}\text { Yield } \\
\text { qt/ha }\end{array}$ & $\begin{array}{c}\text { Saved } \\
\text { yield over } \\
\text { untreated } \\
\text { control }\end{array}$ & $\begin{array}{c}\text { Value of } \\
\text { Saved } \\
\text { yield }\end{array}$ & $\begin{array}{l}\text { Total cost } \\
\text { of } \\
\text { treatment }\end{array}$ & $\begin{array}{c}\text { Net } \\
\text { income } \\
\text { (Rs./ha) }\end{array}$ & $\begin{array}{c}\text { Cost } \\
\text { Benefit } \\
\text { Ratio }\end{array}$ \\
\hline $\mathbf{T}_{1}$ & Spinosad $45 \%$ Sc & $\begin{array}{c}200 \\
\mathrm{ml} / \mathrm{ha}\end{array}$ & 45.01 & 22.81 & 34785.00 & 10440.00 & 24345.00 & $1: 2.33$ \\
\hline $\mathbf{T}_{2}$ & Phorate 10G & $\begin{array}{c}15 \\
\mathrm{~kg} / \mathrm{ha}\end{array}$ & 32.63 & 10.43 & 15905.00 & 4680.00 & 11225.00 & $1: 2.39$ \\
\hline $\mathbf{T}_{3}$ & Carbofuran 3G & $\begin{array}{c}20 \\
\mathrm{~kg} / \mathrm{ha}\end{array}$ & 35.91 & 13.71 & 20907.00 & 5280.00 & 15627.00 & $1: 2.95$ \\
\hline $\mathbf{T}_{4}$ & Bifenthrin $10 \%$ EC & $\begin{array}{c}400 \\
\mathrm{ml} / \mathrm{ha}\end{array}$ & 38.44 & 16.24 & 24766.00 & 2280.00 & 22486.00 & $1: 9.86$ \\
\hline $\mathbf{T}_{5}$ & $\begin{array}{l}\text { Cartap hydrochloride } \\
50 \% \text { SP }\end{array}$ & $1 \mathrm{~kg} / \mathrm{ha}$ & 36.93 & 14.73 & 22463.00 & 4080.00 & 18383.00 & $1: 4.50$ \\
\hline $\mathbf{T}_{6}$ & $\begin{array}{l}\text { Imidachloprid17.8\% } \\
\text { SL }\end{array}$ & $\begin{array}{c}250 \\
\mathrm{ml} / \mathrm{ha}\end{array}$ & 28.41 & 6.21 & 9470.00 & 2325.00 & 7145.00 & $1: 3.35$ \\
\hline $\mathbf{T}_{7}$ & Control & --- & 22.20 & 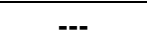 & --- & $\begin{array}{ll}-- \\
\end{array}$ & --- & --- \\
\hline
\end{tabular}

\section{Forty five days after sowing}

The minimum dead heart formation was found in treatment Spinosad 45\% S.C. @ 200 $\mathrm{ml} / \mathrm{ha}$ treated plots (2.33) followed by Bifenthrin $10 \%$ E.C. @ 400 ml/ha (3.33), Cartap hydrochloride 50 \% S.P. @ 1 kg/ha (3.66) and Carbofuran $3 \mathrm{G} @ 20 \mathrm{~kg} / \mathrm{ha}(5.00)$.
The more dead heart were found in treatment Phorate 10 G @ 15 kg/ha (7.00) and Imidachloprid 17.8\% SL @ 250 ml/ha (7.66). Maximum dead hearts was recorded in untreated control (9.33) per cent. (Table no.1) Similarly, Pal et al., (2009), they recorded the damage of $C$. partellus in maize withdead hearts varied from 7.4 to 14.3 percent. 


\section{Leaf injury}

\section{Thirty days after sowing}

The observation showed that the minimum leaf injury (1.33) was found in plots treated with Spinosad 45\% S.C. @ $200 \mathrm{ml} / \mathrm{ha}$ and (1.66) followed by Bifenthrin $10 \%$ E.C. @ $400 \mathrm{ml} / \mathrm{ha}$. Other insecticides, Cartap hydrochloride $50 \quad \% \quad$ S.P. @ $11 \mathrm{~kg} / \mathrm{ha}$, Carbofuran3 G @ 20 kg/ha, Phorate10 G @ $15 \mathrm{~kg} / \mathrm{ha}$ and Imidachloprid 17.8\% SL @ 250 $\mathrm{ml} /$ ha were recorded leaf injury rating in order of (2.78), (4.33), (5.44) and (6.20) leaf injury rating, respectively. The highest leaf injury (7.33) was recorded in the untreated control. (Table no.1)

\section{Forty five days after sowing}

The observation showed that the minimum leaf injury (1.66) was found in the plots treated with Spinosad 45\% S.C. @ $200 \mathrm{ml} / \mathrm{ha}$ followed by Bifenthrin $10 \%$ E.C. @ 400 $\mathrm{ml} / \mathrm{ha}$ (2.33). Other insecticides, Cartap hydrochloride 50 \% $\quad$ S.P. @ 1 kg/ha, Carbofuran3G@20kg/ha, Phorate10 G @ $15 \mathrm{~kg} / \mathrm{ha}$ and Imidachloprid 17.8\% SL @ 250 $\mathrm{ml} /$ ha leaf injury rating were recorded leaf injury rating in order of (3.66), (4.66), (5.66) and (6.66), respectively. The highest leaf injury (8.66) was recorded with untreated control (Table 1). Similarly, Pal et al., (2009) reported that leaf injury rating in kharif shown maize was 2.1 to 7.3 .
Grain yield and cost benefit ratio of different insecticidal treated plots

The maximum mean grain yield was recorded $45.01 \mathrm{q} / \mathrm{ha}$ with Spinosad treatment. Bifenthrin was the second most effective treatment, recorded $38.44 \mathrm{q} / \mathrm{ha}$ of grain yield. The other treatment in descending order were Cartap hydrochloride, Carbofuran, Phorate and Imidachloprid with grain yield of 36.93, $35.91,32.63$ and $28.41 \mathrm{q} / \mathrm{ha}$ (Table 2).

\section{References}

Ahmed, S., Saleem, M. A. and Rauf, I. 2002. Field efficacy of some bioinsecticides against maize and jowar stem borer, Chilo partellus (Pyralidae: Lepidoptera). Journal of Agriculture and Biology; 4(3): 332-334.

James, C., 2003. Global review of Commercialized Transgenic Crops features Bt Maize. ISAAA: Ithaca, New York.

Pal, R., Singh, G., Prasad, C. S., Ali, N., Kumar, A. and Dhaka, S. S. 2009. Field evaluation of Bio-pesticides and Bioagent against stem borer, Chilo partellus (Swinhoe) in Maize. Ann. PI. Protec. Sci., 17(2): 325-327.

Siddiqui, K. H., and K. K. Marwaha, 1993. The vistas of maize entomology in India, Kalyani Publishers, New Delhi, India, 185p.

\section{How to cite this article:}

Vishvendra, Sandeep Kumar, Visvash Vaibhav, Ankush Kumar and Singh, D.V. 2017. Efficacy of Different Management Practices against Chilo partellus (Swinhoe) in Kharif Maize Crop in Western Uttar Pradesh, India. Int.J.Curr.Microbiol.App.Sci. 6(10): 2870-2874. doi: https://doi.org/10.20546/ijcmas.2017.610.337 